\title{
Cosmic-ray theory unravels
}

\section{Astrophysicists ponder whether ultrahigh-energy particles really do come from the centre of galaxies.}

High-energy cosmic rays pack a punch: their particles hold some 10 million times more energy than can be produced by accelerators on Earth. Astronomers had thought that they were getting close to understanding the origins and composition of these intense particles. Now it seems they must think again.

Data collected three years ago by a team at the Pierre Auger Observatory in Mendoza, Argentina, suggested a tentative alignment between these incoming particles and active galactic nuclei (AGN). This hinted that the supermassive black holes in the AGN might be the long-sought cosmic particle accelerators that give the rays their huge energies (The Pierre Auger Collaboration Science 318, 938-943; 2007).

The apparent connection with black holes electrified the astrophysics community. "We thought a new astronomy had been born: charged particle astronomy," says Charles Dermer, a cosmic-ray astrophysicist at the US Naval Research Laboratory in Washington DC.

But at a meeting of the American Physical Society in Washington DC on 16 February, the Auger team began to backpedal on its original conclusions. The group revealed new data that weaken the link between the high-energy particles and the AGN.

Compounding the mystery, the team has found evidence that these highest-energy cosmic rays might be iron nuclei, rather than the protons that make up most cosmic rays. "There are some puzzles," says Paul Sommers, co-spokesperson for the Auger collaboration at Pennsylvania State University in University Park. "We're not close to writing the final chapter."

Low-energy cosmic rays, made mostly of protons, strike Earth continually. They originate within the Milky Way but are seen coming from all directions in the sky because magnetic fields in the Galaxy bend their paths and obscure their original sources. Higher-energy cosmic rays from outside the Galaxy are much less frequent, but are potentially more valuable as astronomical tracers because they barrel into the Galaxy on straighter paths.

The most extreme of them hit the planet with enormous energies of $1 \times 10^{20}$ electronvolts (eV; by comparison, protons produced by the Large Hadron Collider will top out at $7 \times 10^{12} \mathrm{eV}$ ). These high-energy particles could provide a way to detect new physics

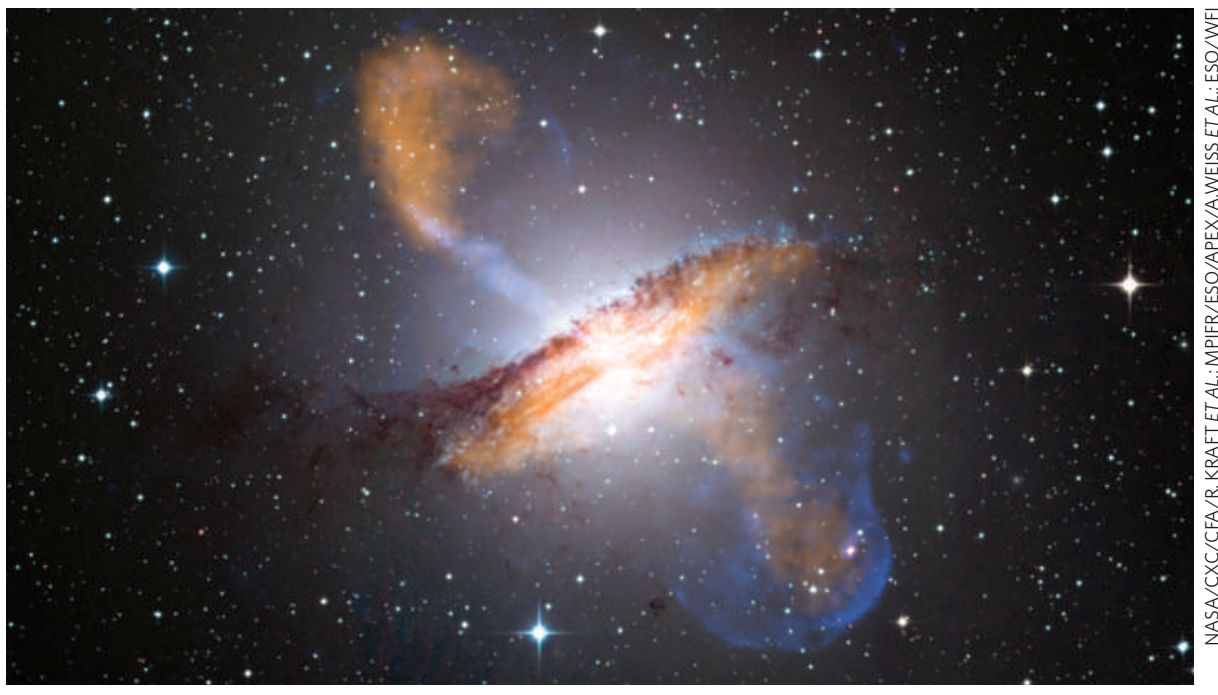

The black hole at the centre of the galaxy Centaurus $A$ is just one potential source of cosmic rays.

beyond the standard model of subatomic particles and the forces that control them. When high-energy particles strike the upper atmosphere, they create 'air showers' of billions of particles and a faint flash of light that, on moonless nights, can be seen at places such as the Auger Observatory, a 3,000-squarekilometre array of detectors on the dry plains of Patagonia.

But gleaning clues about these cosmic rays is difficult because of their rarity; on average, fewer than one particle per century strikes a square kilometre of ground. Over the years, astronomers have considered a handful of possible origins besides the AGN for these rarest of rays. Some suspect the exploding stars that create $\gamma$-ray bursts, and Dermer says that it is possible that even small black holes and neutron stars could ramp the particles up to the necessary energies.

The latest results from the Auger scientists put all of these potential origins back in the game. Although the 2007 paper was based on just 13 of the highest energy cosmic rays, Sommers says that the team is preparing a new analysis for publication that is based on 44 more events. Fewer than $40 \%$ of these cosmic rays seemed to be coming from the AGN, a much weaker correlation than previously reported. The rays are not randomly distributed but follow the distribution of matter visible in the Universe.

The strangest aspect of the latest results relates to the particles' composition. As the team reports in a paper accepted for publication in Physical Review Letters (The Pierre Auger Collaboration, preprint at http://arxiv.org/abs/1002.0699; 2010), they are seeing small air showers that are indicative of iron nuclei, rather than the larger showers that point to protons. Ultrahigh-energy cosmic rays made of iron nuclei would be harder to explain because such nuclei are less common than protons and are more likely to be broken down by the violent mechanisms thought to accelerate cosmic rays.

Complicating the picture further is the fact that a rival experiment in the Northern Hemisphere - the older and smaller High Resolution Fly's Eye (HiRes) experiment in Utah - has produced contradictory data. Spokesman Pierre Sokolsky of the University of Utah in Salt Lake City says that his team's results, based on just ten cosmic rays, suggest that they are protons and come from a random distribution in the sky. The analysis is under review at Astrophysical Journal Letters.

Sokolsky points out that the cosmic-ray flux at HiRes could just be different from that seen in Argentina. Earth's Northern Hemisphere (and therefore HiRes) generally faces away from the centre of the Milky Way, so the magnetic fields in that direction could differ. By contrast, several prominent and nearby potential sources of cosmic rays, such as the AGN of the galaxy Centaurus A, are visible from the Southern Hemisphere. "Nature is throwing us a few curve balls, it looks like," says Sokolsky.

Eric Hand 